\title{
Analysis of Controlling Wireless Temperature Sensor for Monitoring Peat-Land Fire
}

\author{
*Noveri Lysbetti Marpaung \\ Electrical Engineering Department, \\ Universitas Riau \\ Pekanbaru, Indonesia \\ noveri.marpaung@gmail.com \\ Edy Ervianto \\ Electrical Engineering Department, \\ Universitas Riau \\ Pekanbaru, Indonesia \\ edyervianto@eng.unri.ac.id
}

\author{
Rahyul Amri \\ Electrical Engineering Department, \\ Universitas Riau \\ Pekanbaru, Indonesia \\ rahyulamri@eng.unri.ac.id \\ Nurhalim Dani Ali \\ Electrical Engineering Department, \\ Universitas Riau \\ Pekanbaru, Indonesia \\ nurhalim@eng.unri.ac.id
}

*corresponding author: Noveri Lysbetti Marpaung, noveri.marpaung@gmail.com

\begin{abstract}
The problem of forest and peatland fires in Riau has become main topic to prioritize its revamping. Land and forest fires in Riau until reached 3,700 hectares' area, happened in some last years. Forest and peat land fires occurred in the majority of districts in Riau Province, from low category of 4 hectares until 2,800 hectares. This research discusses about analysis of controlling wireless Temperature Sensor for monitoring Peat-Land fire by using LM35 Temperature Sensor, Transmitter-Module (HC-12), ReceiverModule (HC-12), Arduino-ProMini to produce outputs on LED, LCD, Buzzer. Detector of Peat-Land fire works when it is burning, so heat will spread through aluminium stalk and be read by Temperature-Sensor 1 upto Temperature-Sensor4. Output of each sensor is sent to each control block of ArduinoProMini in Transmitter-Module(FU1-FU4). From ArduinoProMini, it is sent to Receiver-Module(FU5). Receiver-Module only receives one data from Transmitter-Module in one time. Every data is received by Receiver-Module goes to ArduinoProMini, processed to produce outputs on LCD that shows ID of FU1-FU4, Peat-Land Status, temperature of Peat Land. If LED is Green, Peat-Land not burned, LED shows SAFE Condition, Buzzer Off. If LED is Yellow, Peat-Land burned underground, LED shows BE-CAREFUL Condition, Buzzer On. If LED is Red, Peat-Land burned on the ground, LED shows DANGER Condition, Buzzer On. Temperature $0^{\circ} \mathrm{C}-$ 21.9 ${ }^{\circ} \mathrm{C}$ is SAFE Category because Peat-Land not burned. Temperature $22^{\circ} \mathrm{C}-28.4^{\circ} \mathrm{C}$ is BE-CAREFUL Category because Peat-Land burned underground. Temperature $28.4^{\circ} \mathrm{C}-50^{\circ} \mathrm{C}$ is DANGER Category because Peat-Land burned on the ground. This equipment works as its frame work.
\end{abstract}

Keywords- LM35 temperature sensor, Microcontroller Arduino pro mini, $\mathrm{HC}-12$ transmitter module, $\mathrm{HC}-12$ receiver module, Output indicators.

\section{INTRODUCTION}

The problem of forest and peatland fires in Riau has become the main topic to prioritize its revamping. Land and forest fires in Riau happened in some last years, until reached an area of 3,700 hectares. Forest and peat land fires occured in the majority of districts in Riau Province, from low category of 4 hectares until the largest reached 2,800 hectares. Fire data recorded at the Riau Forestry may increase in big size.

The conversion of forest areas including peatlands for the development of palm oil, rubber and sago plantation crops will still occur in Riau in the next few years. This directly causes the amount of carbon in the peatlands to be released into emissions if the peatland is converted, drained and flammable. Although various rules and policies have been issued to support the sustainable use of peatlands, these efforts are still insufficient to prevent conversion and emissions. So, more intensive policies and supervision are required [1]

In terms of supervision, there are many ways that can be applied directly. Supervision by human labor will spend much time and energy if the area to be monitored as wide as Riau Province. However, the development of science and technology in the world is one of the answers to priority issues of Peat Land fires.

The objective of this research is to analyze controlling wireless Temperature Sensor for monitoring Peat-Land fire to provides information about condition of peat-land at that moment through LED, LCD and Buzzer. 
Away location of peatlands which is the site of fire, makes it difficult to monitor continuously. With so much peatland, making it difficult to know which place exactly is burned. This makes it difficult for local communities and governments to monitor directly the circumstances of fire. Only by using satellite then can be found the state of hotspot contained in the peat area.

Unfortunately, hotspot location information obtained by satellite is not exactly location where the Peat Land fires occur. This condition is the reason to monitor directly the condition of Peat Land fires by Riau Forestry. Subject matters based on above conditions as follow:

- The range of wireless sensor network transmission is influenced by the area to be served.

- Network topology is selected with consideration in order to reach the transmission distance above 150 m.

- Selection of wireless sensor technology refers to network topology to be selected.

The benefit of this research is to prepare an area is considered vulnerable to peat fires (as early warning system and early detection system), So that fire and smoke haze can be avoided.

\section{LITERATURE REVIEW}

\section{A. Previous Study}

Reference [2] was designed Prototype of Telemetry System Based on Temperature Sensor and Smoke Sensor For Monitoring of Land Fire by using LM35 Sensor and Smoke Sensor type FG200, Microcontroller Atmega 8535, Module RF TXM02 as Transmitter and Module RF TXM01 as Receiver. In this research, Transmitter Module could transmit data with distance $200 \mathrm{~m}$ in open space. Transmitter sent data to Receiver if sensor has sent temperature $37^{\circ} \mathrm{C}$ and or detected smoke. So, buzzer will On and LCD display is sparkling. Data from sensor is also sent through Converter RS323 to display on PC by serial communication.

Reference [3] was Wireless Heat Detector for Monitoring Forest Fire Based on Microcontroller, using data sending system with dengan Frequency Shift Keying (FSK) Module. Temperature Sensor LM35 was used to detect temperature. Result of this research stated that when did testing on temperature $50^{\circ} \mathrm{C}$ could send data to Receiver Module with distance 100meter. This Module detected heat around it. If it detected temperature more than referense $50^{\circ} \mathrm{C}$ so Transmitter Module sent data to Receiver Penerima using wireless system to display on monitor.

Reference [4] did measuring test of 5 samples in Open Space with distance $20 \mathrm{~m}, 40 \mathrm{~m}, 60 \mathrm{~m}, 80 \mathrm{~m}$, and $100 \mathrm{~m}$. Testing result mentioned that the longest distance was $87 \mathrm{~m}$ and used LED as a notification from each data sent.

Reference [5] was title Early Fire Detection System Based on Wireless Sensor Network, stated that the temperature measurements, could be used as a leading indicator to detect early presence of forest fires.
This study combines methods from the previous research. The research uses four Temperature Sensors LM35, Arduino Pro Mini, four HC-12 Transmitter Module and one HC12Receiver Module. Sensors detect any fire of lands, under ground or on the ground. The results of sensors are transmitted by four HC-12 Transmitter Module to HC-12 Receiver Module. Receiver receives just one data for any time. Results of research is displayed on LED, LCD and Buzzer.

\section{B. System of Data Transmitting}

In this research, System of Data Transmitting uses HC12 Module as communication module with serial port by wireless, that can transmit and receive serial data through air media with frequency $433 \mathrm{MHz}$ and distance up to 1800 meter on open space. HC-12 Module is a Data Transmitter and Receiver Data, but it can not transmit and receive data trough air in the same time because link half-duplex only available between Module. This module can be used as a physically cable replacement to substitute communication cable junction of serial half-duplex, that carry TTL signal. So, in its using, must uses two HC-12 Modules, whereas they can be arranged as Data Transmitter and Receiver Data separately [6]. HC-12 Module can be seen in Figure 1.

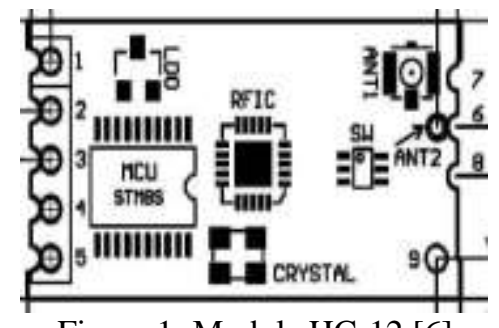

Figure 1: Module HC-12 [6]

Each HC-12 Module works in mode:

- FU1 - Standar Energy Saving Mode with 250000 bps more than air baud rate. Baud rate from serial port can be arranged be any supporting number.

- FU2 - Extrem Energy Saving Mode with 250000 bps, more than air speed. Baud rate from serial port is limited up to $1200 \mathrm{bps}, 2400 \mathrm{bps}, 4800 \mathrm{bps}$.

- FU3 - default, General Objective Mode exceeds different air speed, depend on serial port speed. Equivalent maximum distance such as:

- 1200 bps $\sim 1000 \mathrm{~m}$.

- $2400 \mathrm{bps} \sim 1000 \mathrm{~m}$.

- 4800 bps $\sim 500 \mathrm{~m}$.

- $9600 \mathrm{bps} \sim 500 \mathrm{~m}$.

- 19200 bps $\sim 250 \mathrm{~m}$.

- 38400 bps $~ 250 \mathrm{~m}$.

- $7600 \mathrm{bps} \sim 100 \mathrm{~m}$.

- $115200 \mathrm{bps} \sim 100 \mathrm{~m}$

- FU4 (available on version 2.3 or newest) - Long Distance Mode exceeds air speed which is limited upto 1200 bps because air speed smaller than port speed. Only small packet can be sent like as 60 Byte maximum with interval 2 seconds. This Distance Mode can be raised up to 1800 meter 
Paired HC-12 can create wireless relation work in same mode and with same speed, such as FU1, FU2, FU3, FU4 [7].

\section{Arduino Pro Mini}

Arduino Pro Mini is microcontroller ATMega 328. This Integrated Circuit (IC) has 14 digital pin input/output (whereas six pins can be used as PWM outputs, six analog inputs, resonator on-board, reset button, and hole for header pin. Six header pins can be connected to FTDI cable or Spark fun board breakout to give USB power and communication board [8].

\section{Temperature Sensor LM35}

Temperature LM35 Sensor Module is a temperature sensor can give changing output voltage linearity together with happened changing temperature linearity. LM35Temperature Sensor can operate by using source voltage $4-30 \mathrm{~V}_{\mathrm{DC}}$, to avoid over self heating is used by power supply $5 \mathrm{~V}$. Self heating is heating effect by component itself, consequent of working current through it. Output of LM35 Temperature Sensor is linier scale factor towards the real temperature $10 \mathrm{mV} /{ }^{\circ} \mathrm{C}$, with maximum range can be measured from $-55^{\circ} \mathrm{C}$ upto $150{ }^{\circ} \mathrm{C}$ [9].

Maximum output of LM35 is 1,5 Volt, with accuracy $0.5^{\circ} \mathrm{C}$ on temperature $25^{\circ} \mathrm{C}$. Reading error of sensor on measurement with $25^{\circ} \mathrm{C}$ room temperature but no more $2^{\circ} \mathrm{C}$, can be calculated by using formula in Equation 1.

$\operatorname{Temp}\left({ }^{\circ} \mathrm{C}\right)=V_{\text {out }} m V / 10 \mathrm{Mv}$

\section{MATERIAL \& METHODOLOGY}

\section{A. Data}

In this research, data was collected by measuring of peat land temperature by using LM35 Temperature Sensor which is put on HC-12 Transmitter Module. Four Temperature Sensors (TS1 - TS2) are used to detect land fire. The reading of each sensor is sent to each Microcontroller Arduino Pro Mini (PM1 - PM4) to be processed, before transmit it to HC-12 Receiver Module by each HC-12 Transmitter Module (FU1 - FU4). Only one data can be received in one moment from each FU1 - FU4 to HC-12 Receiver Module. Data from Receiver goes to Microcontroller Arduino Pro Mini (PM5). Then the data is processed to produce output in LED, LCD and Buzzer. Testing is done in Open Space and the Real Condition.

There are three LEDs are used : Green, Yellow and Red. If Green LED ON so, Peat Land Status is Not BURNED, LCD shows SAFE condition, Buzzer OFF. If Yellow LED ON so, Peat Land Status is BURNED condition, LCD shows BE CAREFULL condition, Buzzer ON. If Red LED ON so, Peat Land Status is BURNED condition, LCD shows DANGER condition, Buzzer ON.

Temperature from $0^{\circ} \mathrm{C}$ to $21,9^{\circ} \mathrm{C}$ is SAFE Category because Peat Land is not burned. Temperature from $22^{\circ} \mathrm{C}$ to $28,4^{\circ} \mathrm{C}$ is BE CAREFUL Category because Peat Land started tobe burned underground. Temperature from $28,4^{\circ} \mathrm{C}$ to $50^{\circ} \mathrm{C}$ is DANGER Category because Peat Land burned on the ground

\section{B. Method}

Methods in this research are:

1. Make design of a prototype.

2. Apply designed circuit in a prototype.

3. Measure peat land temperature.

4. Transmit data of Peat Land temperature to Receiver.

5. Reading Peat Land temperature from Receiver will be processed of Microcontroller Arduino ProMini so it can produce outputs on LCD, LED and Buzzer, depend on the value of temperature.

Diagram block in this research, can be seen in Figure 2.

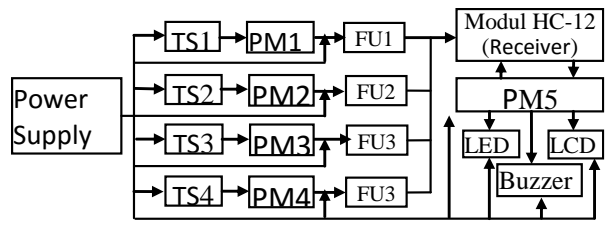

Figure 2: Diagram block of research.

\section{RESULTS AND DISCUSSION}

Testing of Sent Distance Data in Open Space, can be seen in Table 1.

Table 1: Testing of sent distance data in open space

\begin{tabular}{|c|c|c|c|c|}
\hline $\begin{array}{l}\text { Channel } \\
\text { Number }\end{array}$ & Sent Data & $\begin{array}{c}\text { Environmental } \\
\text { Condition }\end{array}$ & $\begin{array}{c}\text { Status of Sent } \\
\text { Data }\end{array}$ & $\begin{array}{l}\text { Distance } \\
\text { (Meter) }\end{array}$ \\
\hline \multirow{6}{*}{ FU1 } & \multirow{6}{*}{$\begin{array}{l}\text { Number } \\
\text { "2"" }\end{array}$} & \multirow{6}{*}{ Open Space } & Received & 50 \\
\hline & & & Received & 100 \\
\hline & & & Received & 150 \\
\hline & & & Received & 200 \\
\hline & & & Received & 250 \\
\hline & & & Not Received & 300 \\
\hline \multirow{11}{*}{ FU2 } & \multirow{11}{*}{$\begin{array}{l}\text { Number } \\
\text { "2" }\end{array}$} & \multirow{11}{*}{ Open Space } & Received & 50 \\
\hline & & & Received & 100 \\
\hline & & & Received & 150 \\
\hline & & & Received & 200 \\
\hline & & & Received & 250 \\
\hline & & & Received & 300 \\
\hline & & & Received & 350 \\
\hline & & & Received & 400 \\
\hline & & & Received & 450 \\
\hline & & & Received & 500 \\
\hline & & & Not Received & 550 \\
\hline \multirow{19}{*}{ FU3 } & \multirow{19}{*}{$\begin{array}{l}\text { Number } \\
\text { "2" }\end{array}$} & \multirow{19}{*}{ Open Space } & Received & 50 \\
\hline & & & Received & 100 \\
\hline & & & Received & 150 \\
\hline & & & Received & 200 \\
\hline & & & Received & 250 \\
\hline & & & Received & 300 \\
\hline & & & Received & 350 \\
\hline & & & Received & 400 \\
\hline & & & Received & 450 \\
\hline & & & Received & 500 \\
\hline & & & Received & 550 \\
\hline & & & Received & 600 \\
\hline & & & Received & 650 \\
\hline & & & Received & 700 \\
\hline & & & Received & 750 \\
\hline & & & Received & 800 \\
\hline & & & Received & 850 \\
\hline & & & Received & 900 \\
\hline & & & Received & 950 \\
\hline
\end{tabular}




\begin{tabular}{|c|c|c|c|c|}
\hline & & & Received & 1000 \\
\hline & & & Not Received & 1050 \\
\hline \multirow{29}{*}{ FU4 } & \multirow{29}{*}{$\begin{array}{l}\text { Number } \\
\text { "2") }\end{array}$} & \multirow{29}{*}{ Open Space } & Received & 50 \\
\hline & & & Received & 100 \\
\hline & & & Received & 150 \\
\hline & & & Received & 200 \\
\hline & & & Received & 250 \\
\hline & & & Received & 300 \\
\hline & & & Received & 350 \\
\hline & & & Received & 400 \\
\hline & & & Received & 450 \\
\hline & & & Received & 500 \\
\hline & & & Received & 550 \\
\hline & & & Received & 600 \\
\hline & & & Received & 650 \\
\hline & & & Received & 700 \\
\hline & & & Received & 750 \\
\hline & & & Received & 800 \\
\hline & & & Received & 850 \\
\hline & & & Received & 900 \\
\hline & & & Received & 950 \\
\hline & & & Received & 1000 \\
\hline & & & Received & 1100 \\
\hline & & & Received & 1200 \\
\hline & & & Received & 1300 \\
\hline & & & Received & 1400 \\
\hline & & & Received & 1500 \\
\hline & & & Received & 1600 \\
\hline & & & Received & 1700 \\
\hline & & & Received & 1800 \\
\hline & & & Not Received & 1850 \\
\hline
\end{tabular}

From Table 1, can be seen that sent distance data by Transmitter in Open Space has many categories due to Channel Number. Channel 1 (FU1) can send data from distance $50 \mathrm{~m}-250 \mathrm{~m}$. Sent data can be read/received well in this range by Receiver Module. If distance exceeds 250m, so data cannot be transmitted or received.

For Channel 2 (FU2) can transmit data from $50 \mathrm{~m}-$ $500 \mathrm{~m}$. Transmitted data can be read/received well in this range by Receiver Module. If distance exceeds 500m, so data cannot be transmitted or received.

On Channel 3 (FU3) can send data from distance 50m$1000 \mathrm{~m}$. Sent data can be read/received well in this range by Receiver Module. If distance exceeds $1000 \mathrm{~m}$, so data cannot be transmitted or received.

Channel 4 (FU4) can send data from distance 50m $1800 \mathrm{~m}$. Transmitted data can be read/received well in this range by Receiver Module. If distance exceeds $1800 \mathrm{~m}$, so data cannot be transmitted or received.

Testing of Sent Distance Data in real condition, is shown Table 2.

Table 2: Sent distance data in real condition

\begin{tabular}{|c|c|c|c|c|}
\hline $\begin{array}{l}\text { Channel } \\
\text { Number }\end{array}$ & Sent Data & $\begin{array}{c}\text { Environmental } \\
\text { Condition }\end{array}$ & $\begin{array}{c}\text { Status of } \\
\text { Sent Data }\end{array}$ & $\begin{array}{l}\text { Distance } \\
\text { (Meter) }\end{array}$ \\
\hline \multirow{4}{*}{ FU1 } & \multirow{4}{*}{ Number "2" } & \multirow{4}{*}{$\begin{array}{l}\text { Experimental } \\
\text { Test : } \\
\text { - Buildings } \\
\text { - Trees }\end{array}$} & Received & 50 \\
\hline & & & Received & 100 \\
\hline & & & Received & 130 \\
\hline & & & Not Received & 140 \\
\hline \multirow{7}{*}{ FU2 } & \multirow{7}{*}{ Number "2" } & \multirow{7}{*}{$\begin{array}{l}\text { Experimental } \\
\text { Test : } \\
\text { - Buildings } \\
\text { - Trees }\end{array}$} & Received & 50 \\
\hline & & & Received & 100 \\
\hline & & & Received & 150 \\
\hline & & & Received & 200 \\
\hline & & & Received & 250 \\
\hline & & & Received & 270 \\
\hline & & & Not Received & 280 \\
\hline FU3 & Number "2" & Experimental & Received & 50 \\
\hline
\end{tabular}

\begin{tabular}{|c|c|c|c|c|}
\hline & & \multirow{10}{*}{$\begin{array}{l}\text { Test : } \\
\text { - Buildings } \\
\text { - Trees }\end{array}$} & Received & 100 \\
\hline & & & Received & 150 \\
\hline & & & Received & 200 \\
\hline & & & Received & 250 \\
\hline & & & Received & 300 \\
\hline & & & Received & 350 \\
\hline & & & Received & 400 \\
\hline & & & Received & 450 \\
\hline & & & Received & 460 \\
\hline & & & Not Received & 470 \\
\hline \multirow{19}{*}{ FU4 } & \multirow{19}{*}{ Number " 2 " } & \multirow{19}{*}{$\begin{array}{l}\text { Experimental } \\
\text { Test : } \\
\text { - Buildings } \\
\text { - Trees }\end{array}$} & Received & 50 \\
\hline & & & Received & 100 \\
\hline & & & Received & 150 \\
\hline & & & Received & 200 \\
\hline & & & Received & 250 \\
\hline & & & Received & 300 \\
\hline & & & Received & 350 \\
\hline & & & Received & 400 \\
\hline & & & Received & 450 \\
\hline & & & Received & 500 \\
\hline & & & Received & 550 \\
\hline & & & Received & 600 \\
\hline & & & Received & 650 \\
\hline & & & Received & 700 \\
\hline & & & Received & 750 \\
\hline & & & Received & 800 \\
\hline & & & Received & 850 \\
\hline & & & Received & 870 \\
\hline & & & Not Received & 880 \\
\hline
\end{tabular}

From Table 2, can be seen that sent distance data by Transmitter in Real Condition on Experimental Test with Buildings and Trees, have many categories due to Channel Number. Channel 1 (FU1) can transmit data from 50m $130 \mathrm{~m}$. Transmitted data can be received well in this range by Receiver Module. If distance exceeds 130m, so data cannot be transmitted or received.

For Channel 2 (FU2) can send data from 50m-270m. Sent data can be received well in this range by Receiver Module. If distance exceeds $270 \mathrm{~m}$, so data cannot be transmitted or received.

On Channel 3 (FU3) can transmit data from 50m -460m. Transmitted data can be received well in this range by Receiver Module. If distance exceeds $460 \mathrm{~m}$, so data cannot be transmitted or received.

Channel 4 (FU4) can send data from 50m - 870m. Sent data can be received well in this range by Receiver Module. If distance exceeds $870 \mathrm{~m}$, so data cannot be transmitted or received.

Testing of Tool totally for DANGER Category, can be seen in Table 3 .

Table 3: Testing for tool totally for danger category

\begin{tabular}{|c|c|c|c|c|c|c|c|c|}
\hline \multirow{2}{*}{$\begin{array}{c}\text { Environ- } \\
\text { mental } \\
\text { Condition }\end{array}$} & \multirow{2}{*}{$\begin{array}{c}\text { Distan- } \\
\text { ce } \\
\text { (Meter) }\end{array}$} & \multicolumn{3}{|c|}{ Display of LCD } & \multicolumn{3}{|c|}{ LED } & \multirow[b]{2}{*}{ Buzzer } \\
\hline & & ID & $\begin{array}{l}\text { Tempera } \\
\text { ture }\left({ }^{\circ} \mathrm{C}\right)\end{array}$ & Category & Green & Yellow & $\operatorname{Red}$ & \\
\hline \multirow{13}{*}{$\begin{array}{l}\text { Experimental } \\
\text { Test: } \\
\text { - Buildings } \\
\text { - Trees }\end{array}$} & \multirow{4}{*}{50} & FU1 & 28,2 & Burned & - & - & $\sqrt{ }$ & ON \\
\hline & & FU2 & 28,7 & Burned & - & - & $\sqrt{ }$ & $\mathrm{ON}$ \\
\hline & & FU3 & 29,3 & Burned & - & - & $\sqrt{ }$ & $\mathrm{ON}$ \\
\hline & & FU4 & 29,9 & Burned & - & - & $\sqrt{ }$ & $\mathrm{ON}$ \\
\hline & \multirow{4}{*}{100} & FU1 & 28,2 & Burned & - & - & $\sqrt{ }$ & $\mathrm{ON}$ \\
\hline & & FU2 & 28,7 & Burned & - & - & $\sqrt{ }$ & $\mathrm{ON}$ \\
\hline & & FU3 & 29,3 & Burned & - & - & $\sqrt{ }$ & $\mathrm{ON}$ \\
\hline & & FU4 & 29,9 & Burned & - & - & $\sqrt{ }$ & $\mathrm{ON}$ \\
\hline & \multirow{4}{*}{140} & FU1 & 28,2 & Burned & - & - & $\sqrt{ }$ & $\mathrm{ON}$ \\
\hline & & FU2 & 28,7 & Burned & - & - & $\sqrt{ }$ & $\mathrm{ON}$ \\
\hline & & FU3 & 29,3 & Burned & - & - & $\sqrt{ }$ & $\mathrm{ON}$ \\
\hline & & $\overline{\text { FU4 }}$ & 29,9 & Burned & - & - & $\sqrt{ }$ & $\mathrm{ON}$ \\
\hline & 150 & FU1 & - & - & - & - & $\sqrt{ }$ & OFF \\
\hline
\end{tabular}




\begin{tabular}{|c|c|c|c|c|c|c|c|}
\hline & FU2 & 28,7 & Burned & - & - & $\sqrt{ }$ & $\mathrm{ON}$ \\
\hline & FU3 & 29,3 & Burned & - & - & $\sqrt{ }$ & ON \\
\hline & FU4 & 29,9 & Burned & - & - & $\sqrt{ }$ & ON \\
\hline \multirow{4}{*}{200} & FU1 & - & - & - & - & $\sqrt{ }$ & OFF \\
\hline & $\overline{F U 2}$ & 28,7 & Burned & - & - & $\sqrt{ }$ & ON \\
\hline & FU3 & 29,3 & Burned & - & - & $\sqrt{ }$ & ON \\
\hline & FU4 & 29,9 & Burned & - & - & $\sqrt{ }$ & ON \\
\hline \multirow{4}{*}{250} & FU1 & - & - & - & - & $\sqrt{ }$ & OFF \\
\hline & FU2 & 28,7 & Burned & - & - & $\sqrt{ }$ & ON \\
\hline & $\overline{\text { FU3 }}$ & 29,3 & Burned & - & - & $\sqrt{ }$ & $\mathrm{ON}$ \\
\hline & $\overline{\text { FU4 }}$ & 29,9 & Burned & - & - & $\sqrt{ }$ & ON \\
\hline \multirow{4}{*}{270} & FU1 & - & - & - & - & $\sqrt{ }$ & OFF \\
\hline & FU2 & 28,7 & Burned & - & - & $\sqrt{ }$ & ON \\
\hline & $\overline{\text { FU3 }}$ & 29,3 & Burned & - & - & $\sqrt{ }$ & ON \\
\hline & $\overline{\text { FU4 }}$ & 29,9 & Burned & - & - & $\sqrt{ }$ & ON \\
\hline \multirow{4}{*}{300} & FU1 & - & - & - & - & $\sqrt{ }$ & OFF \\
\hline & FU2 & - & - & - & - & $\sqrt{ }$ & OFF \\
\hline & FU3 & 29,3 & Burned & - & - & $\sqrt{ }$ & $\mathrm{ON}$ \\
\hline & $\overline{\text { FU4 }}$ & 29,9 & Burned & - & - & $\sqrt{ }$ & ON \\
\hline \multirow{4}{*}{350} & FU1 & - & - & - & - & $\sqrt{ }$ & OFF \\
\hline & FU2 & - & - & - & - & $\sqrt{ }$ & OFF \\
\hline & FU3 & 29,3 & Burned & - & - & $\sqrt{ }$ & ON \\
\hline & FU4 & 29,9 & Burned & - & - & $\sqrt{ }$ & ON \\
\hline \multirow{4}{*}{400} & FU1 & - & - & - & - & $\sqrt{ }$ & OFF \\
\hline & FU2 & - & - & - & - & $\sqrt{ }$ & OFF \\
\hline & $\overline{\text { FU3 }}$ & 29,3 & Burned & - & - & $\sqrt{ }$ & ON \\
\hline & FU4 & 29,9 & Burned & - & - & $\sqrt{ }$ & ON \\
\hline \multirow{4}{*}{450} & FU1 & - & - & - & - & $\sqrt{ }$ & OFF \\
\hline & FU2 & - & - & - & - & $\sqrt{ }$ & OFF \\
\hline & FU3 & 29,3 & Burned & - & - & $\sqrt{ }$ & ON \\
\hline & FU4 & 29,9 & Burned & - & - & $\sqrt{ }$ & ON \\
\hline \multirow{4}{*}{460} & FU1 & - & - & - & - & $\sqrt{ }$ & OFF \\
\hline & FU2 & - & - & - & - & $\sqrt{ }$ & OFF \\
\hline & FU3 & 29,3 & Burned & - & - & $\sqrt{ }$ & ON \\
\hline & FU4 & 29,9 & Burned & - & - & $\sqrt{ }$ & $\mathrm{ON}$ \\
\hline \multirow{4}{*}{500} & FU1 & - & - & - & - & $\sqrt{ }$ & OFF \\
\hline & FU2 & - & - & - & - & $\sqrt{ }$ & OFF \\
\hline & FU3 & - & - & - & - & $\sqrt{ }$ & OFF \\
\hline & FU4 & 29,9 & Burned & - & - & $\sqrt{ }$ & ON \\
\hline & FU1 & - & - & - & - & $\sqrt{ }$ & OFF \\
\hline 550 & $\overline{\text { FU2 }}$ & - & - & - & - & $\sqrt{ }$ & OFF \\
\hline 550 & $\overline{\text { FU3 }}$ & - & - & - & - & $\sqrt{ }$ & OFF \\
\hline & FU4 & 29,9 & Burned & - & - & $\sqrt{ }$ & ON \\
\hline & FU1 & - & - & - & - & $\sqrt{ }$ & OFF \\
\hline 600 & FU2 & - & - & - & - & $\sqrt{ }$ & OFF \\
\hline 600 & $\overline{\text { FU3 }}$ & - & - & - & - & $\sqrt{ }$ & OFF \\
\hline & $\overline{\text { FU4 }}$ & 29,9 & Burned & - & - & $\sqrt{ }$ & $\mathrm{ON}$ \\
\hline & FU1 & - & - & - & - & $\sqrt{ }$ & OFF \\
\hline 650 & FU2 & - & - & - & - & $\sqrt{ }$ & OFF \\
\hline 650 & FU3 & - & - & - & - & $\sqrt{ }$ & OFF \\
\hline & $\overline{\text { FU4 }}$ & 29,9 & Burned & - & - & $\sqrt{ }$ & ON \\
\hline & FU1 & - & - & - & - & $\sqrt{ }$ & OFF \\
\hline 700 & FU2 & - & - & - & - & $\sqrt{ }$ & OFF \\
\hline 100 & FU3 & - & - & - & - & $\sqrt{ }$ & OFF \\
\hline & FU4 & 29,9 & Burned & - & - & $\sqrt{ }$ & $\mathrm{ON}$ \\
\hline & FU1 & - & - & - & - & $\sqrt{ }$ & OFF \\
\hline 750 & FU2 & - & - & - & - & $\sqrt{ }$ & OFF \\
\hline 150 & FU3 & - & - & - & - & $\sqrt{ }$ & OFF \\
\hline & FU4 & 29,9 & Burned & - & - & $\sqrt{ }$ & ON \\
\hline & FU1 & - & - & - & - & $\sqrt{ }$ & OFF \\
\hline 800 & $\overline{F U 2}$ & - & - & - & - & $\sqrt{ }$ & OFF \\
\hline 800 & $\overline{\text { FU3 }}$ & - & - & - & - & $\sqrt{ }$ & OFF \\
\hline & FU4 & 29,9 & Burned & - & - & $\sqrt{ }$ & ON \\
\hline & FU1 & - & - & - & - & $\sqrt{ }$ & OFF \\
\hline 850 & FU2 & - & - & - & - & $\sqrt{ }$ & OFF \\
\hline 850 & $\overline{\text { FU3 }}$ & - & - & - & - & $\sqrt{ }$ & OFF \\
\hline & $\overline{\text { FU4 }}$ & 29,9 & Burned & - & - & $\sqrt{ }$ & ON \\
\hline & FU1 & - & - & - & - & $\sqrt{ }$ & OFF \\
\hline 870 & FU2 & - & - & - & - & $\sqrt{ }$ & OFF \\
\hline $8 / 0$ & FU3 & - & - & - & - & $\sqrt{ }$ & OFF \\
\hline & FU4 & 29,9 & Burned & - & - & $\sqrt{ }$ & ON \\
\hline 000 & FU1 & - & - & - & - & $\sqrt{ }$ & OFF \\
\hline 900 & FU2 & - & - & - & - & $\sqrt{ }$ & OFF \\
\hline
\end{tabular}

\begin{tabular}{|l|c|c|c|c|c|c|c|c|}
\hline & & FU3 & - & - & - & - & $\sqrt{ }$ & OFF \\
\cline { 2 - 7 } & & FU4 & - & - & - & - & $\sqrt{ }$ & OFF \\
\hline
\end{tabular}

From Table 3, can be seen that temperature reading $28,2^{\circ} \mathrm{C}$ from Temperature Sensor1 (FU1) with data sent distance from 50m-270m, shows BURNED Category, Red LED and Buzzer are ON.

Temperature reading $28,7^{\circ} \mathrm{C}$ from Temperature Sensor2 (FU2) with data sent distance from 50m- 270m, shows BURNED Category, Red LED and Buzzer are ON.

Temperature reading $29,3^{\circ} \mathrm{C}$ from Temperature Sensor3 (FU3) with data sent distance from 50 meter - 270 meter, shows BURNED Category, Red LED and Buzzer are ON.

Temperature reading $29,9^{\circ} \mathrm{C}$ from Temperature Sensor 4 (FU4) with data sent distance from 50m- 270m, shows BURNED Category, Red LED and Buzzer are ON.

Based on result in Table 3, so Peat Land Status is in DANGER Condition.

\section{CONCLUSION}

For Open Space, temperature Sensor1 (FU1) can transmit data from $50 \mathrm{~m}-250 \mathrm{~m}$, Temperature Sensor2 (FU2) can send data from $50 \mathrm{~m}-500 \mathrm{~m}$, temperature Sensor3 (FU3) can transmit data from $50 \mathrm{~m}-1000 \mathrm{~m}$, temperature Sensor4 (FU4) can send data from $50 \mathrm{~m}-1800$ $\mathrm{m}$.

For Real Condition on Experimental Test such as buildings and trees, Temperature Sensor1 (FU1) can send data from $50 \mathrm{~m}-130 \mathrm{~m}$, Temperature Sensor2 (FU2) can transmit data from $50 \mathrm{~m}-270 \mathrm{~m}$, Temperature Sensor3 (FU3) can send data from $50 \mathrm{~m}-460 \mathrm{~m}$, Temperature Sensor4 (FU4) can transmit data from $50 \mathrm{~m}-870 \mathrm{~m}$.

If Green LED ON so, Peat Land Status is Not BURNED, LCD shows SAFE condition, Buzzer OFF. If Yellow LED ON so, Peat Land Status is BURNED condition, LCD shows BE CAREFULL condition, Buzzer ON. If Red LED ON so, Peat Land Status is BURNED condition, LCD shows DANGER condition, Buzzer ON.

Temperature from $0{ }^{\circ} \mathrm{C}$ to $21,9{ }^{\circ} \mathrm{C}$ is SAFE Category because Peat Land is not burned. Temperature from $22{ }^{\circ} \mathrm{C}$ to $28.4{ }^{\circ} \mathrm{C}$ is BE CAREFUL Category because Peat Land started to be burned underground. Temperature from 28,4 ${ }^{\circ} \mathrm{C}$ to $50{ }^{\circ} \mathrm{C}$ is DANGER Category because Peat Land burned on the ground.

\section{REFERENCES}

[1] Research Centre Riau Province, "Research of Peat Land Fire Preventif System Trough Electromagnetic Reflection Wave Prediction in Riau Province", Research Centre Riau Province, 2014.

[2] Lapanporo, Boni Pahlanop, "Prototipe of Telemetry System Based on Temperature Sensor and Smoke Sensor For Monitoring of Land Fire", Journal Positron Pontianak: University Tanjungpura Vol. 1 No. 1, pp $43-49,2011$.

[3] R. Amri, "Detector of Wireless Heat For Monitoring of Jungle Firing Based on Microcontroller", Journal of Sainstek Vol. 2 No. 2, pp 40, 2014.

[4] Muamar, Taufik, "Design Power Supply Management System on Early Warning System Node For Disaster Mitigation of Peat Land Fire", Electrical Engineering, Universitas Riau, 2017.

[5] Haryawan, M.Y., Gunawan, A, "Early Fire Detection System Based on Wireless Sensor Network", Teknik Informasi dan Telematik Journal, Vol. 1, No. 1, pp. 04-05, 2014.

[6] Elecrow, "HC-12 Wireless Serial Port Communication Module," https://www.elecrow.com, 2012. 
[7] Dziku, "HC-12 433MHz Wireless Serial Communication Module Configuration”, Electronics Lab, https://quadmeup.com, 2016.
[8] Arduino Team, "Arduino Pro Mini, Spark Fun Electronics", https://creativecommons.org, 